\title{
Heat transfer coefficient determination for flow boiling in vertical and horizontal minichannels
}

\author{
Magdalena Piasecka ${ }^{1, a}$, Beata Maciejewska ${ }^{1}$ \\ ${ }^{1}$ Kielce University of Technology, Al. 1000-lecia P.P. 7, 25-314 Kielce, Poland
}

\begin{abstract}
The paper presents the results of boiling heat transfer research during FC-72 laminar flow along a minichannel of $1 \mathrm{~mm}$ depth, positioned vertically and horizontally, with an enhanced heating surface. One glass pane allows to determine the temperature of the heating wall by liquid crystal thermography. Calculations are aimed at the evaluation of one- and two-dimensional heat transfer approaches to determine the local heat transfer coefficient. In the one-dimensional approach only the direction of the flow in the channel is considered. In the two-dimensional approach the inverse problem in the heating wall and the direct problem in the glass barrier were solved by the finite element method with Trefftz functions as shape functions (FEMT). The developed flow boiling area was studied. Heat transfer coefficient values obtained for the horizontal minichannel were higher than those obtained for the vertical one. When the heat flux supplied to heating wall grows, the share of gas-phase increases leading to the heat transfer coefficient decreases. The same courses of the experiment were observed for the two applied methods, but the results obtained in the one-dimensional approach are considerably higher than in the two-dimensional one. One-dimensional approach seems to be less sensitive to measurement errors.
\end{abstract}

\section{Introduction}

Boiling is an extremely efficient heat transfer process used in power generation, chemical industry and nuclear engineering. One of the relevant boiling features is the high value of the heat transfer coefficient, due to which large heat fluxes can be transported. Miniheat exchangers are used to provide higher cooling capability for new technologies. Owing to the change of state, which accompanies flow boiling in minichannels, it is possible to meet contradictory demands simultaneously, i.e. to obtain a heat flux as large as possible at small temperature difference between the heating surface and the saturated liquid and, at the same time, retain small dimensions of heat transfer systems. Review of relevant literature and the selected publications covering flow boiling heat transfer in minichannels is presented in $[1,2]$, and having enhanced surfaces - in [3-5]. It leads to the conclusion that although much has been written recently on flow boiling heat transfer in minichannels, the observations related to the effects of various factors on boiling heat transfer in minichannels are diverse and frequently conflicting. They are usually verified experimentally for channel systems heated by smooth heating surfaces.
The studies concentrating on enhanced structure systems attract attention due to their use in their theoretical enhancement potential for heat transfer.

\section{Experimental issues}

\subsection{Experimental test module}

The main part of the experimental stand is the test module with a minichannel (figure $1 \mathrm{~b}, \# 1$ ) oriented vertically (position $90^{\circ}$, figure 1a) and horizontally (position $0^{\circ}$, figure $1 \mathrm{~b}$ ). It is $1 \mathrm{~mm}$ deep, $40 \mathrm{~mm}$ wide and $360 \mathrm{~mm}$ long. The heating element for the working fluid (FC-72) flowing laminarly along the minichannel is 0.1 mm Haynes-230 alloy foil (\#2). The foil on the side of the fluid flowing in the minichannel is single-sided enhanced, with micro-recesses distributed evenly (\#5). The opposite side of the heating foil is covered with thermosensitive liquid crystals (\#3) and piece of glass (\#4b). The application of liquid crystals thermography allows to observe changes in the hue of the foil surface (figure 1c) and on that basis to determine the temperature distribution (figure 2). Another glass pane (\#4a) allows to observe two-phase flow structures. Thermocouples

\footnotetext{
$\overline{{ }^{a} \text { Corresponding author: tmpmj@tu.kielce.pl }}$
} 
K-type (\#7) and pressure converters (\#6) are installed on the inlet and outlet of the minichannel. The investigations focus on developed boiling.

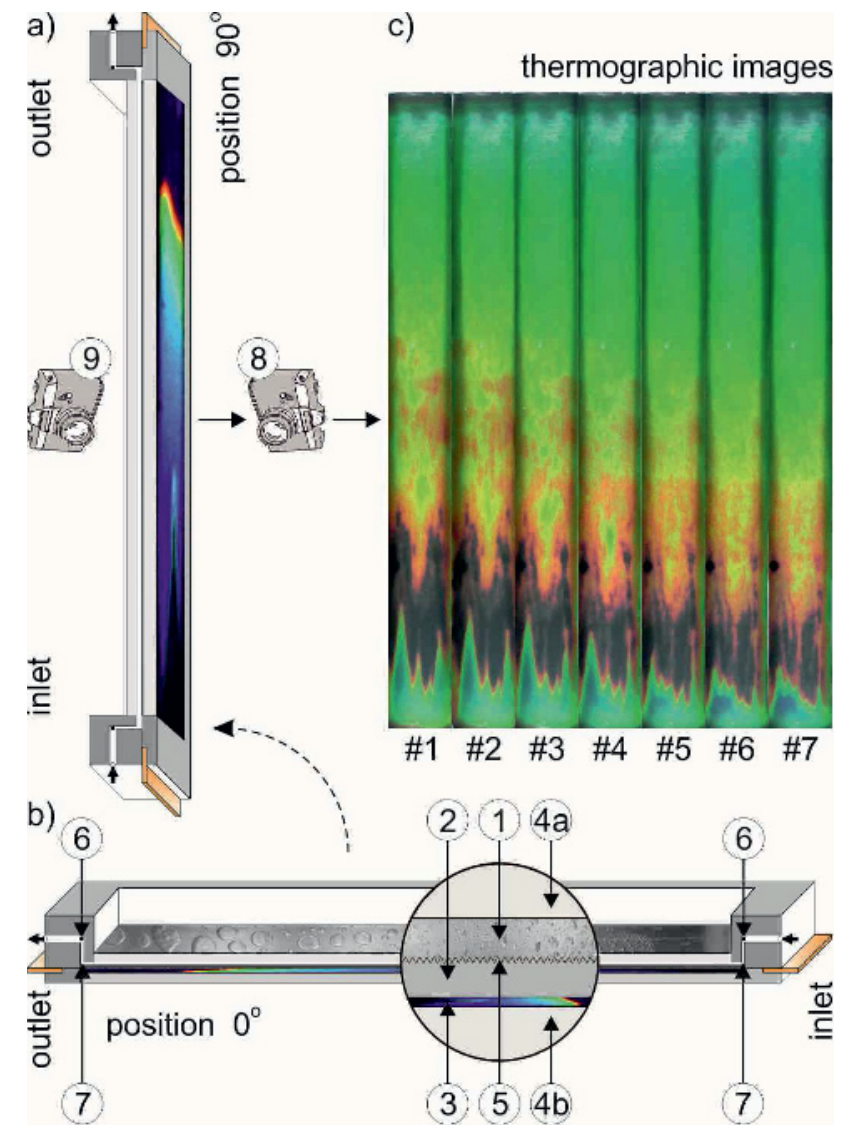

Figure 1. a,b) The schematic diagrams of the test module: a) vertical orientation, position $90^{\circ}$ : \#1-minichannel, \#2-heating foil, \#3-liquid crystal layer, \#4a,b-glass pane, \#5-enhanced surface, \#6-pressure converter, \#7-thermocouple, \#8-digital camera, \#9-digital SLR camera; b) horizontal orientation, position $0^{\circ}$; c) thermographic images of the heating foil with liquid crystals for minichannel positioned vertically, experimental parameters: flow velocity $0.12 \mathrm{~m} / \mathrm{s}$, mass flux $212 \mathrm{~kg} \mathrm{~m}^{-2} \mathrm{~s}^{-1}$, pressure at the inlet to the channel $140 \mathrm{kPa}$, inlet liquid subcooling $43 \mathrm{~K}$; volumetric heat flux $q_{v}=1.84+10^{5} \div 2.29+10^{5} \mathrm{~kW} \mathrm{~m}^{-3}$.

The experimental stand was presented in detail in [2-6]. Apart from the test module the flow loop consists of the rotary pump, the compensating tank, the heat exchanger, the filter, rotameters and the deaerator. The data and the image acquisition system is composed of: digital cameras $(\# 8, \# 9)$, the data acquisition station, a computer with special software and lighting systems.

The micro-recesses on the heating foil were made by laser drilling. They are evenly distributed every $100 \mu \mathrm{m}$ in both axes. The diameter of the single micro-recesses is $10 \mu \mathrm{m}$, its depth is $3 \mu \mathrm{m}$. A 5-7 $\mu \mathrm{m}$ high layer of melted metal accumulates annularly around the recesses, forming the structure of "craters", figure 2.

The accuracy of heating foil temperature measurements by using liquid crystal thermography and heat source efficiency measurement error in the same research set up were evaluated in [2,7]. The mean temperature measurement error of heating foil by liquid crystal thermography was $0.86 \mathrm{~K}$. The relative volumetric heat flux measurement error amounted to $3.53 \%$.
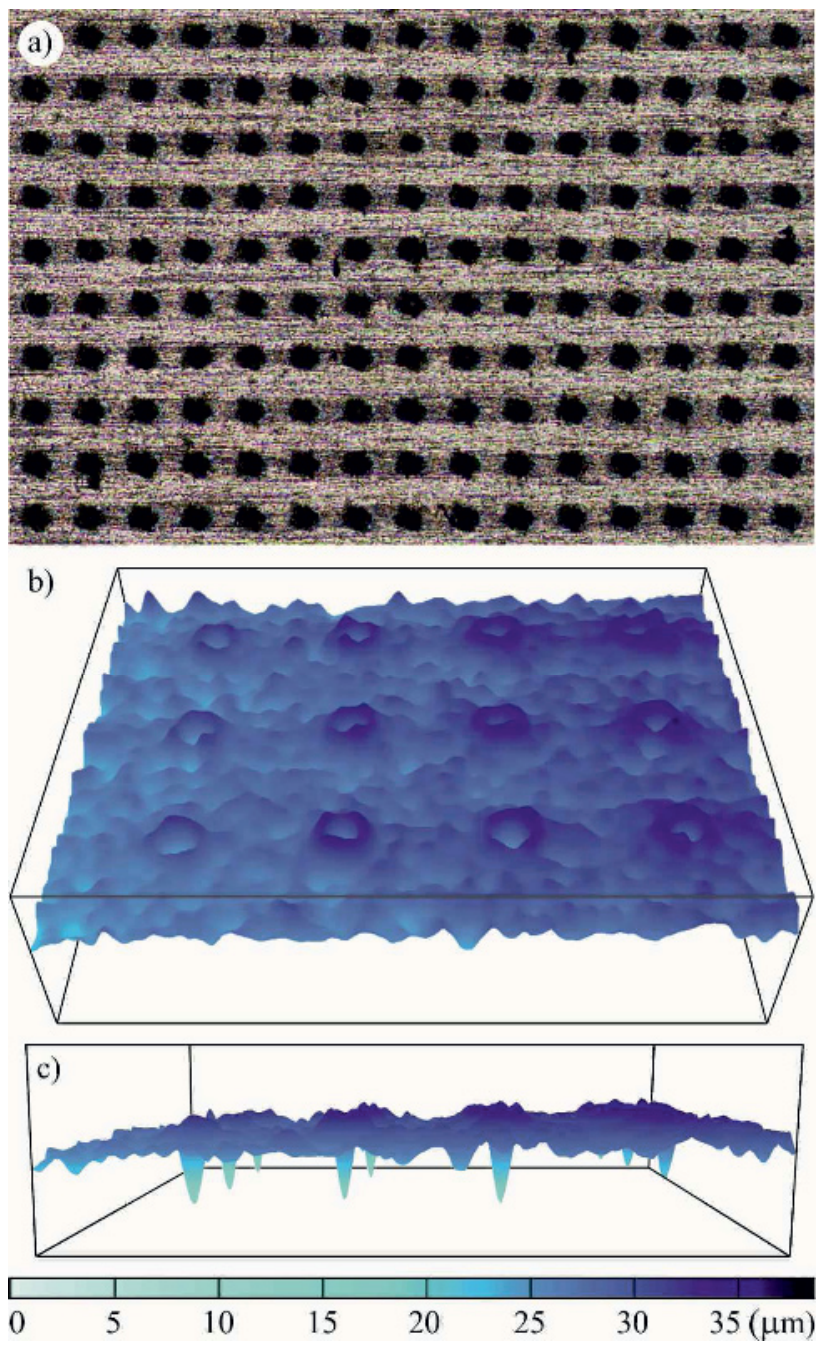

Figure 2. Photo (a), 3D topography (b) and a fragment of a cross-section of the sampled area of the enhanced foil with micro-recesses.

\subsection{Experimental procedure}

Application of the liquid crystal thermography for the detection of two-dimensional heating surface temperature distribution must be preceded by colour (hue) temperature calibration [2,7]. After deaeration, there is a laminar flow of the working fluid (FC-72) in the main loop of the experimental stand. When the desired pressure and flow rate are reached, the gradual increase in the electric power supplied to the heating foil results in an increased heat flux transferred to the liquid in the minichannel. This leads to the incipience and then to the development of nucleate boiling. Thanks to the liquid crystal layer located on its surface contacting the glass it is possible to measure temperature distribution on the heating wall. Flow structure is observed simultaneously in the opposite side of the minichannel. Measurements are recorded for the assumed heat flux supplied to the minichannel's heating surface, in stable thermal and flow conditions, thus the results relate to steady regimes. 


\section{Mathematical problem}

\subsection{Problem formulation}

The aim of the study is to identify local values of the heat transfer coefficient between the enhanced heating foil and the fluid in the minichannel during flow boiling. This coefficient is determined from the third kind condition. The thickness of liquid crystal layers and temperature changes in glass, foil and liquid along the minichannel width are omitted. Application of liquid crystal thermography provides information on the temperature of the heating foil adjacent to the glass. It is assumed that the external surface of the glass barrier is isolated thermally.

The study is limited to the identification of heat transfer coefficient in saturated flow boiling. The issue of determination of heat transfer coefficient during the onset of boiling flow boiling was discussed in $[1,2,4]$.

\subsection{One-dimensional approaches}

The objective of the calculations is to evaluate one- and two-dimensional heat transfer approaches to calculate the heat transfer coefficient. Two simple one-dimensional approaches [1] give similar results. One approach based on the equations of the energy balance for individual control volumes of the test section. It took into account the dimension along the flow direction $-x$, the dimension perpendicular to it was related only to foil or glass thickness. According to the other approach, local heat transfer coefficient for the saturated flow boiling could be obtained from the equation:

$$
\alpha_{1}(x)=\frac{I \cdot U / A_{F}}{T_{F}(x)-T_{s a t}(x)-\frac{I \cdot U}{A_{F}} \cdot \frac{\delta_{F}}{\lambda_{F}}}
$$

where $I$-current, $U$-voltage drop, $A_{F}$-foil surface, $\lambda_{F}$-foil thermal conductivity, $\delta_{F}$-foil thickness, $T_{F}$-foil temperature, $T_{\text {sat }}$-saturation temperature determined on the basis of linear pressure distribution, from the inlet to the outlet.

\subsection{Two-dimensional approaches}

Papers $[1,2,4,8]$ presented various methods of the determination of the local heat transfer coefficient in the two-dimensional approaches, obtained from solving the inverse problem, amongst others, by the finite element method, the sensitivity coefficient method or the nodeless Trefftz method.

In two-dimensional approaches the temperature $T_{F}$ of the heating foil is determined by solving an inverse problem for heat conductivity:

$$
\nabla^{2} T_{F}=-\frac{q_{V}}{\lambda_{F}} \text { for }(x, y) \in \Omega_{F}
$$

where $\Omega_{F}=\left\{(x, y) \in R^{2}: x_{1}<x<x_{P}, \delta_{G}<y<\delta_{G}+\delta_{F}\right\}$

$$
\begin{gathered}
T_{F}\left(x, \delta_{G}\right)=T_{G}\left(x, \delta_{G}\right) \text { for } x_{1}<x<x_{P} \\
\lambda_{F} \frac{\partial T_{F}}{\partial y}=\lambda_{G} \frac{\partial T_{G}}{\partial y} \text { on } y=\delta_{G} \text { for } x_{1}<x<x_{P} \\
T_{F}\left(x_{1}, y\right)=T_{1} \text { for } \delta_{G}<y<\delta_{G}+\delta_{F} \\
T_{F}\left(x_{P}, y\right)=T_{P} \text { for } \delta_{G}<y<\delta_{G}+\delta_{F} \\
T_{F}\left(x_{p}, \delta_{G}\right)=T_{p} \text { for } p=1,2, \ldots P
\end{gathered}
$$

where $x_{1}$ is the location of the primary temperature measurement on the boundary $y=\delta_{G}, x_{P}$ location of the last temperature measurement, $T_{p}$ - value of temperature measurement, $P$ - number of temperature measurements, $\delta_{G}$ thickness of the glass barrier, $\delta_{F}$ thickness of the heating foil.

The auxiliary temperature $T_{G}$ of the glass barrier is obtained by the solution of the direct problem:

$$
\nabla^{2} T_{G}=0 \text { for }(x, y) \in \Omega_{G}
$$

where $\Omega_{G}=\left\{(x, y) \in R^{2}: 0<x<L, \quad 0<y<\delta_{G}\right\}$

$$
\begin{gathered}
\frac{\partial T_{G}}{\partial y}=0 \text { on } y=0 \text { for } 0<x<L \\
\frac{\partial T_{G}}{\partial x}=0 \text { on } x=0, x=L \text { for } 0<y<\delta_{G} \\
T_{G}\left(x_{p}, \delta_{G}\right)=T_{p} \text { for } p=1,2, \ldots P
\end{gathered}
$$

In order to solve problems under consideration, areas $\Omega_{G}, \Omega_{F}$ are divided into rectangular elements $\Omega_{G}^{j}$ for $j=1,2, \ldots, K_{G}$ and $\Omega_{F}^{j}$ for $j=1,2, \ldots, K_{F}$, nodes are established and then base functions (shape functions) are constructed in the form of linear combinations of Trefftz functions.

Base functions are constructed thanks to following property in nodes of elements $\Omega_{G}^{j}$ or $\Omega_{F}^{j}$ :

$$
\varphi_{j k}\left(x_{i}, y_{i}\right)=\delta_{k i}, \quad i=1,2, \ldots, l w
$$

where: $j$ denotes the element number, $k$ is the base function number in $j$ th element; $l w$ is the number of nodes in the element, $\delta_{k i}$ - Kronecker delta,

$$
\delta_{k i}= \begin{cases}1 & k=i \\ 0 & k \neq i\end{cases}
$$

Temperature approximation in any element $\Omega_{G}^{j}$ is presented as a linear combination of obtained base functions $\varphi_{j k}(x, y)$ :

$$
\widetilde{T}_{G}^{j}(x, y)=\sum_{k=1}^{l w} \hat{T}_{G}^{n} \varphi_{j k}(x, y)
$$

where: $n$ is the node number in the whole area $\Omega_{G}, \hat{T}_{G}^{n}$ is the sought temperature value in the $n$th node of area $\Omega_{G}$, 
while $j, k$ and $l w$ have the same meanings as in formula (12). Unknown coefficients $\hat{T}_{G}^{n}$ in linear combination (14) are determined by the minimization of the appropriate functional which expresses a mean square error of the approximate solution on domain boundary and along common edges of neighbouring elements [4]. Foil temperature is determined by a similar method. In any element $\Omega_{F}^{j}$ it is presented as a linear combination of base functions $\varphi_{j k}(x, y)$ :

$$
\widetilde{T}_{F}^{j}(x, y)=u(x, y)+\sum_{k=1}^{l w} \varphi_{j k}(x, y)\left(\hat{T}_{F}^{n}-u\left(x_{n}, y_{n}\right)\right)
$$

where $u(x, y)$ - the particular solution of equation (2), $n$ is the node number in the whole area $\Omega_{F}, u\left(x_{n}, y_{n}\right)-$ value of the particular solution in the $n$th node of the area $\Omega_{F}, \hat{T}_{F}^{n}$ is the sought value of the temperature in the $n$th node of area $\Omega_{F}$, while $j, k$ and $l w$ have the same meanings as in formula (12). Unknown coefficients $\hat{T}_{F}^{n}$ in linear combination (15) are determined by the minimization of the appropriate functional.

In two-dimensional approaches local values of the heat transfer coefficient were calculated from the formula:

$-\lambda_{F} \frac{\partial T_{F}\left(x, \delta_{G}+\delta_{F}\right)}{\partial y}=\alpha_{2}(x)\left(T_{F}\left(x, \delta_{G}+\delta_{F}\right)-T_{s a t}(x)\right)$

where $\alpha_{2}$ is the sought heat transfer coefficient, while $T_{\text {sat }}(x)$ is the saturation temperature determined on the basis of linear pressure distribution, from the inlet to the outlet.

The following function was assumed as a particular solution of equation (2):

$$
u(x, y)=-\frac{q_{V} y^{2}}{2 \lambda_{F}}
$$

In the finite element method, area $\Omega_{G}$ was divided into 350 rectangular elements. The division of area $\Omega_{F}$ is dependent on the number and location of the measurement points. In setting 18 connected with minichannel oriented horizontally the number of elements is the highest and equals 188. In setting 14 connected with minichannel oriented vertically the number of elements is the lowest and it is 100 . In each rectangular element $\Omega_{G}^{j}$, a system of four nodes located at the vertices of the element was constructed. The measurement points occurring in condition (7) are located in relevant nodes of the grid.

The base functions are the combination of the four Trefftz functions : $1, x, y, x y$.

The results of heat transfer coefficients obtained by presented methods are showed in figure $5 \mathrm{~b}$ and figure. $6 \mathrm{~b}$.
The mean relative error $\sigma$ of heat transfer coefficients was determined in the same way as in the article [4].

Tables 1 and 2 present mean relative errors of the heat transfer coefficient calculated by means FEMT.

Table 1. Mean relative errors of the heat transfer coefficient obtained by FEMT in minichannel oriented vertically.

\begin{tabular}{|c|c|}
\hline Set No. & $\sigma[\%]$ \\
\hline$\# 1$ & 30.3 \\
\hline$\# 2$ & 31.3 \\
\hline$\# 3$ & 32.1 \\
\hline$\# 4$ & 31.9 \\
\hline$\# 5$ & 31.6 \\
\hline$\# 6$ & 31.1 \\
\hline$\# 7$ & 28.7 \\
\hline
\end{tabular}

Table 2. Mean relative errors of the heat transfer coefficient obtained by FEMT in minichannel oriented horizontally.

\begin{tabular}{|c|c|}
\hline Set No. & $\sigma[\%]$ \\
\hline$\# 1$ & 40.3 \\
\hline$\# 2$ & 34.7 \\
\hline$\# 3$ & 32.9 \\
\hline$\# 4$ & 33.1 \\
\hline$\# 5$ & 36.6 \\
\hline$\# 6$ & 36.2 \\
\hline$\# 7$ & 37.9 \\
\hline$\# 8$ & 32.9 \\
\hline$\# 9$ & 35.3 \\
\hline
\end{tabular}

\section{Results}

\subsection{Local foil temperature and heat transfer coefficients}

Thermographic images of the heating foil with liquid crystals for the vertical minichannel are presented in figure $1 \mathrm{c}$ and for the horizontal minichannel - in figure 4a.

Resulting local heating foil temperature dependences on the distance along the minichannel length for the analysed series with the vertical and horizontal minichannel are shown in figure 3 and figure $4 \mathrm{~b}$, respectively.

Heat transfer coefficient dependences determined from one- and two-dimensional approaches, on the distance along the minichannel length, are presented in figure 5 for the vertical minichannel and in figure 6 - for horizontal minichannel. Figures labeled (a) refer to the data obtained for one-dimensional approach, labeled (b) for two-dimensional approach. In addition, the figures contain data for the whole range and, for clarity of data, data for lower values heat transfer coefficients are presented below in each graph for the narrow data range. 


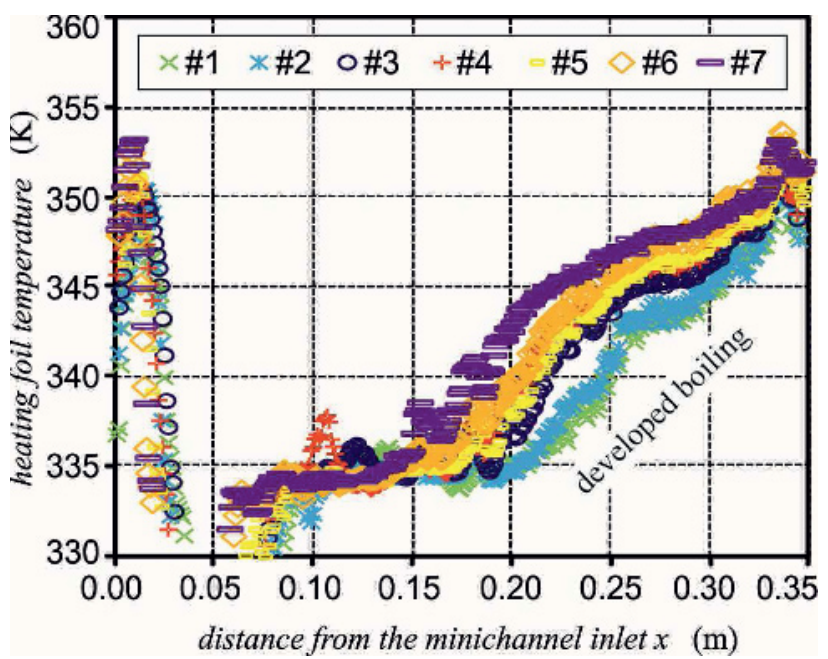

Figure 3. Heating foil temperature dependence on the distance along the minichannel length, experimental data as for figure $1 \mathrm{c}$.

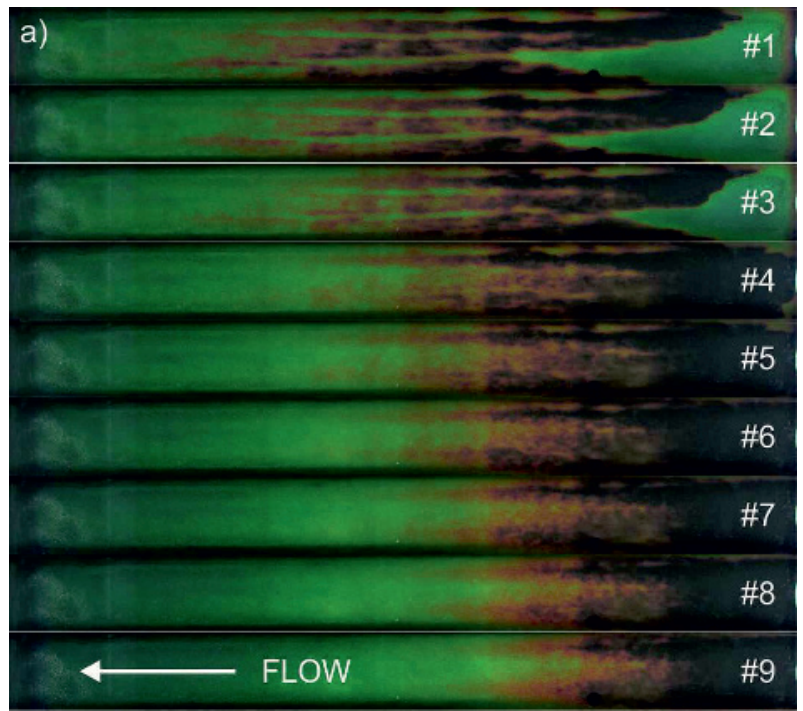

b)

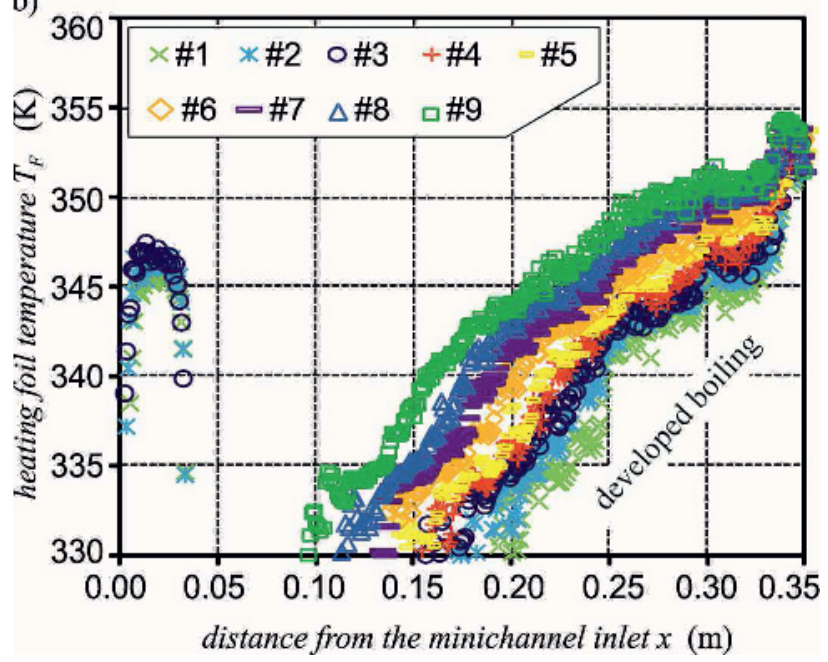

Figure 4. Data for minichannel positioned horizontally

a) thermographic images of the heating foil with liquid crystals, b) heating foil temperature dependence on the distance along the minichannel length, experimental parameters: flow velocity $0.12 \mathrm{~m} \mathrm{~s}^{-1}$, mass flux $212 \mathrm{~kg} \mathrm{~m}^{-2} \mathrm{~s}^{-1}$, pressure at the inlet to the channel $140 \mathrm{kPa}$, inlet liquid subcooling $43 \mathrm{~K}$; volumetric heat flux $q_{v}=1.61+10^{5} \div 2.26 \cdot 10^{5} \mathrm{~kW} \mathrm{~m}{ }^{-3}$.
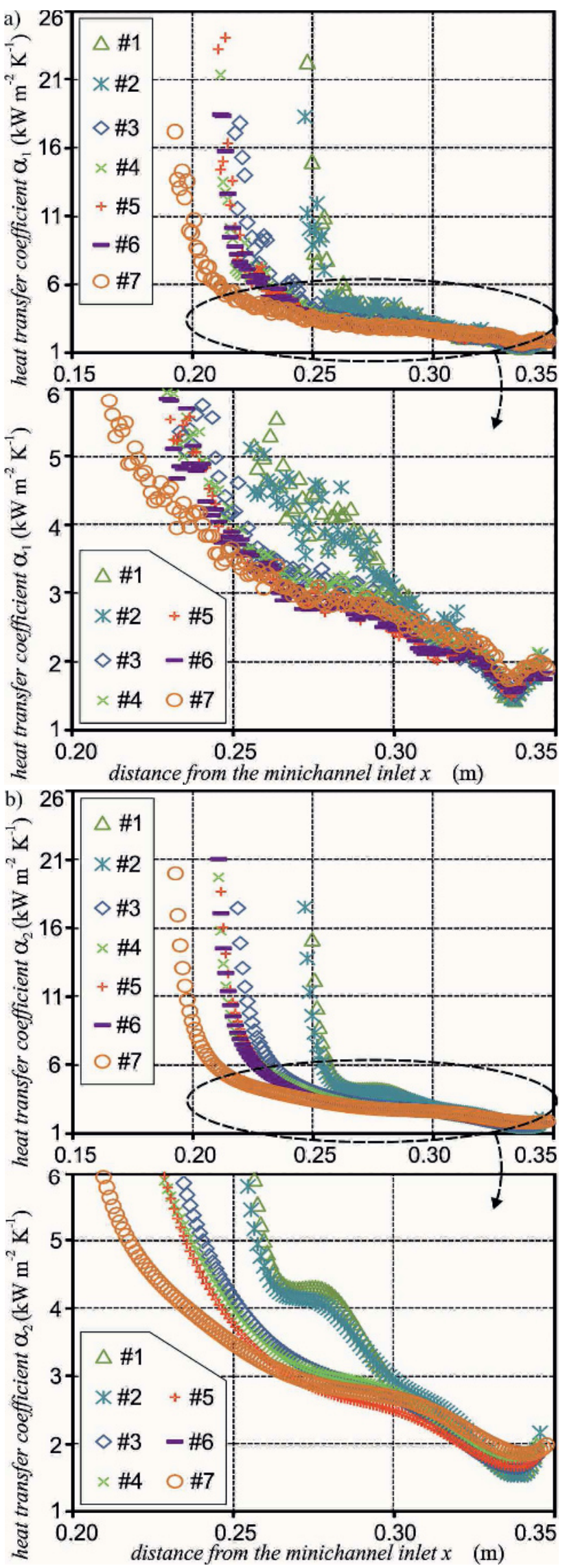

Figure 5. Heat trancfer coefficient dependences on the distance along the minichannel length determined from one- (a) and twodimensional approach (b), minichannel oriented vertically, experimental data as for figure 1. 

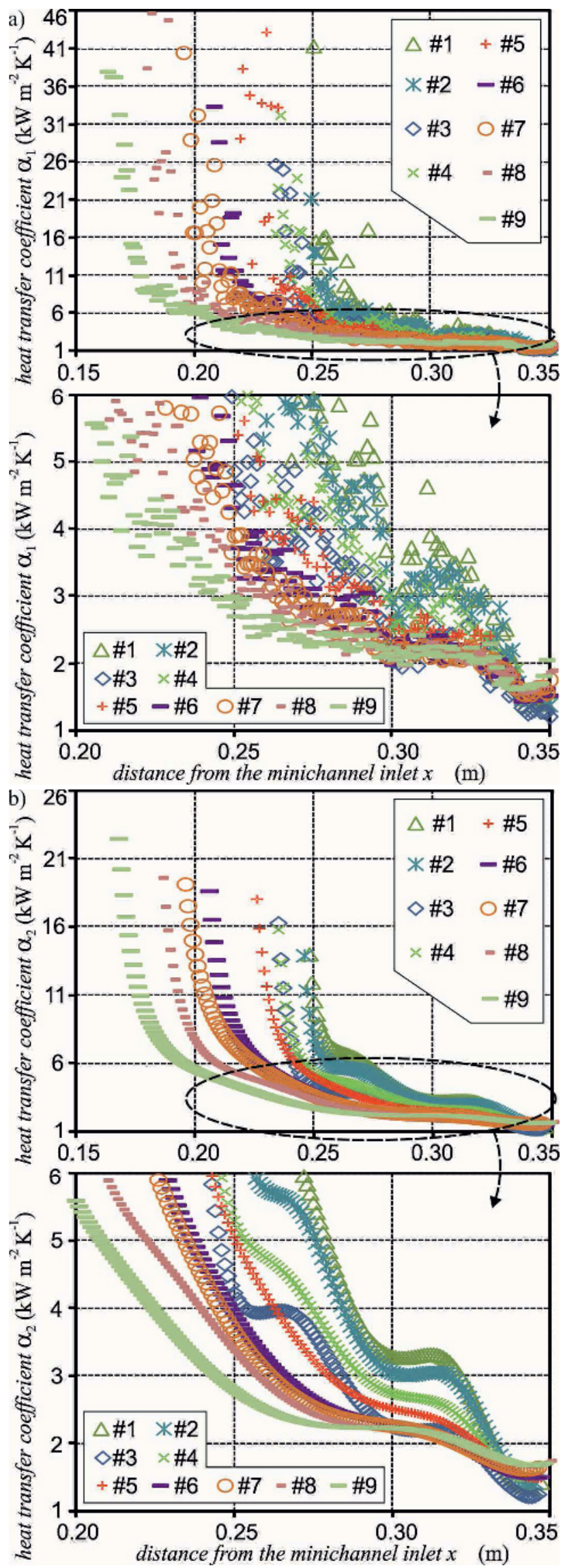

Figure 6. Heat transfer coefficient dependences on the distance along the minichannel length determined from one- (a) and two-dimensional approach (b), minichannel oriented horizontally (fluid above the heating wall), experimental data as for figure 4 .

\subsection{Data analysis}

The analysis was conducted on the basis of data shown in Figs. 3-6. Initially, when the heat flux increases in experimental conditions, the hue sequence in the lower part of images is specific to the "boiling front" accompanying boiling incipience during subcooled boiling (figure 1c, figure $4 \mathrm{a}$, setting from \#1 to \#3). However, this issue is not the subject of this article and it was discussed in detail in [1-6].

In the further part of the experiment when the heat flux continues to increase, a hue sequence in the upper part of images appears (figure 1c, figure 4a). The issue of saturated nucleate boiling, discussed here, is accompanied by an increase in foil temperature, which is observed in the outlet to the minichannel. This occurs in the progress of the developed nucleate boiling in the minichannel. A number of associated phenomena accompany this process: pressure increase in the channel, a sharp increase in the fluid temperature of the flow core and flow resistance fluctuations. In developed nucleate boiling, region local heat transfer coefficients decrease for higher shares of the vapour phase in the two-phase mixture (see Figs. 5 and 6 with magnifications - data taken directly from the minichannel outlet).

Dependence analysis for local heat transfer coefficients, shown in figure 5 (for vertical minichannel) and figure 6 (for horizontal minichannel) presents higher values of the heat transfer coefficients obtained from the horizontally positioned minichannel. Simultaneously, the same course was observed for the two selected calculation methods, while values of the heat transfer coefficient for a low heat flux supplied to the heating surface are similar. However, the results of the onedimensional approach for the last settings at the maximum heat flux supplied to the heating surface (for the fully developed flow boiling) occurred to be considerably higher than in the case of the twodimensional approach. Calculation methods have a big impact and the results differ when this method is applied. Generally, simplified one-dimensional approach, seems to be less sensitive to measurement errors in comparison with the two-dimensional one. Temperature measurement of the heating surface by liquid crystal thermography is burdened with a high error when the red colour of the surface is observed (the lowest temperatures of the active range of liquid crystal mixture). Besides, superheating $T_{F}-T_{\text {sat }}$ of the heating surface appearing in eq. (1) in the one-dimensional approach reaching values close to unity, causes a considerable increase in local heat transfer coefficient values which are sometimes rejected manually in calculations. In the two-dimensional approach, manual operations are not possible. Heating surface superheating close to unity is set a constant value which results from the temperature measurement by liquid crystals thermography. Such points are automatically rejected in the numerical and it may lead to underevaluation of maximum heat transfer coefficient values obtained by this method. 


\section{Conclusions}

The paper presents the results of boiling heat transfer research during FC-72 through a minichannel of $1 \mathrm{~mm}$ depth with an enhanced heating surface, positioned vertically and horizontally. The objective of the calculations is to evaluate one- and two-dimensional heat transfer approaches to calculate the heat transfer coefficient. In the one-dimensional approach the direction along the flow in channel is considered. In the twodimensional approach both the inverse problem in heating foil and the direct problem in glass barrier were solved by means the finite element method with Trefftz functions as shape functions (FEMT). Data limited to the developed flow boiling area was analysed. The results were presented as thermographic images of the heating surface and local heat transfer coefficients. As the heat flux supplied to heating foil grows, the share of gas-phase increases which results in heat transfer coefficient drop. Moreover, higher local heat transfer coefficient values obtained for the horizontally positioned minichannel in comparison with the vertical orientation were recorded. The same kind of the distribution was observed for the two applied methods, however for the measurement data from developed flow boiling area are considerably higher than in the two-dimensional approach. One-dimensional approach seems to be less sensitive to measurement errors.

\section{References}

1. S. Hożejowska, M. Piasecka, M.E. Poniewski, Int. J. Therm. Sci. 48, 1049-1059 (2009)

2. M. Piasecka, B. Maciejewska, Exp. Thermal Fluid Sci. 38, 19-32 (2012)

3. M. Piasecka, Int. J. Heat and Mass Transf. 66, 472488 (2013)

4. M. Piasecka, B. Maciejewska, Exp. Thermal Fluid Sci. 44, 23-33 (2013)

5. M. Piasecka, Heat Mass Transf. 49, 261-271 (2013)

6. M. Piasecka, Metrology and Meas. Sys. XX, 205216 (2013)

7. M. Piasecka, Exp. Heat Transfer, DOI: 10.1080/08916152.2013.782374, in print (2013)

8. S. Hożejowska, R. Kaniowski, M.E. Poniewski, Int. J. Numer. Methods Heat Fluid Flow, manuscript ID: HFF-01-2013-0022.R2, accepted for publication in 2013 